\title{
Ontology based KM: A Review
}

\author{
Rajiv Kumar" \\ Department of Science \& Technology, \\ Govt. of India and \\ PhD Research Scholar, Jodhpur National \\ University, Jodhpur
}

\author{
MD Tiwari \\ Indian Institute of Information Technology, \\ Allahabad, India
}

\begin{abstract}
Knowledge management helps organizations to exploit and develop resources, improve their competitiveness and develop sustainable competitive advantage. Main focus of the research in $\mathrm{KM}$ is on theoretical concepts, approaches, and sustaining tools for human knowledge management. KM plans to manage knowledge by organizing formal and direct method to manage organizational knowledge in the workplace. There are many literatures that have shown a number of KM approaches developed with the purpose of managing organizational knowledge. However, there is a need to focus on study of ontology based collaborative filtering in the existing business environment. This paper reviews ontology-based KM approaches. The primary goal of this work is to understand and evaluate each approach by analyzing and using.
\end{abstract}

\section{Keywords}

Ontology, Knowledge Management (KM), Review

\section{INTRODUCTION}

Organized information regarding the products, markets and technologies are used to constitute the knowledge assets of a company. Knowledge enhances the value at different levels in an organization namely strategic, tactical and organization. The activity of $\mathrm{KM}$ is very wide and complex processing which can be employed over a long period of time. KM has lots of practices to perform with human relationships as it performs with Information Technology and Business practices. It may be the management of individual knowledge or the management of enterprise knowledge. It also includes activities that outline the communication of implicit knowledge to the integration of explicit knowledge. The actions involved in KM process are Knowledge Gathering, Knowledge Organization and Structuring, Knowledge Refinement, and Knowledge Distribution. In recent years, this has been observed that KM has turn into a vital sensation factor for business practices.

Ontology (a discipline of philosophy) provides a semantic skeleton in many applications for KM. It divides the knowledge about things according to their essential persona and represents the hierarchical structuring for them. Use of the concepts of ontology can improve the functioning of search engines [1], ontology acts as the prime functionality in the area of KM. Traditionally, it represents the shared concepts in a domain as in the case of Artificial Intelligence through hierarchical description for the terms which is being used, thereby, leveraging the collaboration and integration of tools [2]. However, ontology based KM is not so easy to sustain and requires an expansion phase.

The primary goal of this survey is understanding and evaluation of each tool by analyzing and using them. This paper is organized as follows. Section 2 lists some goals and motivations about the ontology in KM. Section 3 gives a survey for ontology-based KM approaches. The proposed work is discussed in Section 4 and the conclusions are discussed in Section 5.

\section{GOALS AND MOTIVATION}

Knowledge is an asset in the world nowadays. Common management systems and applications support such knowledge structures. Therefore the key to develop a workable solution for KM lies with setting up the electronic repository of intellectual capabilities of stakeholders. Such repository forms the basis for a Skills Management System and can be used to expose skill gaps and competency levels, to permit the search for people with precise talents and skills, and can manipulate the requirements for training, education and learning opportunities [3]. One can distinguish numerous factors motivating the Ontology in KM.

- Knowledge discovery is a main problem in present digital library system. Semantic digital libraries give extended facilities for knowledge discovery and mechanisms for linking and searching related concepts.

- Ontologies are a primary tool in Artificial Intelligence (AI) in developing the Knowledge-Based Systems. With its formal and well defined structure ontology provides a machine-understandable language that allows automatic reasoning for problems resolution.

- Ontology design, structure and organization are the fundamental importance for the development of a KM System.

- With the help of questionnaires and assessments, individual knowledge could be accessed and problems and barriers of management could be tackled with the chosen ontology-based approach.

- The use of ontology-based KM over long period, consistency and usability can be preserved, and thus change and evolution becomes visible to the user.

- The development and purpose of an ontology model enables semantic modelling and storage of contentand context-oriented information about media items.

Ontologies is useful to retrieve the information from documents, ensure consistency and correctness by constraints on the content of information, integrate the information from various sources, create libraries of interchangeable and reusable models and support inference to derive additional knowledge from facts.

\section{ONTOLOGY IN KM}

$\mathrm{KM}$ is used to represent, organize, create usage, and evaluate knowledge in its many forms. To build effective technologies for $\mathrm{KM}$, further understanding is needed on how individuals, and/or organizations use knowledge. Also, Ontology can be applied to KM at various stages i.e., for knowledge retrieval, storage and sharing [4]. Since this concept uses a hierarchical 
based search for the important concepts and terms in a domain, therefore it is more efficient when compared to the keyword based search method which uses semantics functionality. This ontology finds its relevance throughout the ontology life cycle which includes creation, population, implementation and maintenance of ontologies [5].

Kougias et al. [6] has contributed to reduce the conceptual gap existing between the perception of the instructor and the learner working on a subject using the semantics involved in their cognitive profile by means of ontologies and its operations. It has been observed that to solve the issues of Next Generation Education Environments (NGEEs), there are two necessary operations of ontology to be considered; ontology merging and ontology alignment. In merger a new ontology is created using two or more source ontologies. Here, the output has been created by replacing the source ontology and containing the knowledge captured from the same, since it uses two or more ontologies for knowledge capturing. Therefore, it contains highly overlapping or identical domains. This is put to use when the focus is on defining a domain with greater accuracy. The other option of alignment relates to describe the semantic links between two ontologies without any alterations made to the original ones.

Further they intend to focus on technical details of building the web-based application by adding the proposed ontologies, aiming at dynamically supporting the instructor to plan each and every lesson taking into consideration the semantic links between the Class Profile and the Instructor Profile ontologies.

In [7], the authors proposed a model for the exploitation of ontology-based Knowledge Base to improve search over large document repositories and to provide better search capabilities which yield a qualitative improvement over keyword-based full-text search. The authors introduced finer grained domain ontologies. The proposed system enables detailed as well as topic wise search, as per the classification in the available knowledge base. To manage large size information, a classic vector space model has been proposed upon which a ranking algorithm has been defined. The result of performance test of the proposed model is directly proportional to the amount and quality of information present in the Knowledge Base. Though efforts have and are being made in the area of automating ontology population and text annotation but there still exists a limitation on the completeness of available ontology and Knowledge base. This situation indicates that the techniques of keyword based search and practice of recall will prevail till the time ontology information is unavailable or incomplete.

For managing the ontology based KM system the approach used by the authors, Stojanovic \& Stojanovic [8], analyses the user's behaviour in two phases of a KM cycle. In the first phase, knowledge is provided by carefully examining the comments or meta attached with the content, the second phase analyse the queries generated by the user and corresponding response from the knowledge base [9]. This analyses results in recommendations for the ontology being used, annotations and the knowledge repository to improvise the searching mechanism. Besides this the approach also lays down the guidelines based on the changes in users need, which is to be followed to avoid overloading of knowledge and removing the gaps in the system.

Barnaghi et al. [11] investigated various concepts for creating ontology -based information search and retrieval system, which are: the different techniques of ontology development, ontology assemblage from different data sources, complex and relevant relationship between the data along with their ranking and presentation, social network analysis and presentation methodology. The approach adopted by the author emphasizes on a framework which shows the interdependence of semantic annotation, indexing and retrieval of documents. This annotation framework consists of two components: a light weight upper level ontology and a strong knowledge base. Light weight ontology has several advantages such as it is easy to understand, expand, verify and maintain. In comparison to the traditional approaches for search and retrieval, semantic and ontology based method has an upper hand owing to its flexibility in incorporating query denotation, context based exploration, enhanced data integration, query expansion and most importantly it is more precise and enjoys better recalling mechanism.

Aviv \& Gal [12] developed an application for language transfer option used for multilingual portability based on the Users need. The research work depends on ontology based model for the multilingual language transfer process. To solve the particular problem they used a system that is build to support multilingual ontology management and allows an ontology search to be performed retrieving documents that related to a specific ontology concept. The system have unique features like as lightweight mechanism, dubbed context that is associated with ontological concept and specified in multiple languages. In such applications, the development of an information system that is monolingual will result in low portability and high deployment costs that is a major problem of the research. The solution of the particular problem is to use translation and multilingual information retrieval process. However, translation of language have some specific difficulties, such as the importance of the connection between grammar and meaning, the role of word endings and word position, and the length and complexity of words, which are comprised of other words. To solve the problem they present a solution that bypasses machine translation in multilingual environments by using a single ontology system to which predetermined manually translated ontology concepts are automatically mapped. The solution is multilingual information retrieval process that is based on the multilingual ontological management. They also used a model named as multilingual information system. The information system works in the process as selection, collection, extraction, and adaptation. In the Selection process, the existing ontology is selected. In the next step (i.e. Collection process), the sample document, representing Ontology concepts, are collected. Then the contexts are extracted from the sample documents in the next step and, finally, in the Adaptation step, extracted contexts are associated with ontology concepts.

As huge amount of valuable information available on Web, it is very hard and time consuming to retrieve thousands of web pages related to a concept, filter the relevant ones, analyze this information and integrate it in a knowledge repository. Banares - Alcantara et al. [13], in their article, emphasized on how to retrieve thousands of web pages, filter and analyze the information and integrates it in a knowledge repository. To solve the Research problem, they used a system named as MASH (Multi Agent Search Engine). MASH is able to detect web pages related to domain ontology and calculate the relevance of each one. In MASH four different types of agents were identified: one user Agent (UA), which interacts with the user, second, internet agents (IA) which access, retrieves, rates and filters the information from the web, third, weight agent (WA) that supports the search and supplies alternative 
queries when requested, and Fourth, coordinator agent (CA) that rules the overall process, particularly during splitting and amalgamation of the tasks performed by the IA. Finally both the input and the output of the search process are handled using a web interface where the user can edit, create, retrieve, import and export ontology as Resource Description Format files.

Jan Paralic and Ivan Kostial in their paper [14] mentioned that the ontology-based approach to information retrieval (IR) is based on a domain knowledge representation schema in the form of ontology. To solve the problem they developed package with three different approaches to document retrieval: vector representation, latent semantic indexing method (LSI), and ontology -based method used in the Webocrat system. They stated that the Vector Representation Approach is based on vector representation of document collection. In the beginning, every document is passed through a set of preprocessing tools. The pre-processing tools includes lower case, stop words, document frequency etc. LSI approach is derived from singular value decomposition of tf-idf matrix. Three matrixes are computed by this decomposition. Ontology-Based Approach describes the Webocrat-like approach that uses ontology for document retrieval purposes. This research work left lot of enthusiastic amongst scientific community for future work focused on enhancement of ontology-based retrieval mechanism using more sophisticated inference mechanism for finding similar concepts to given query.

Jarraret et al. [15] developed a highly generic system which offers exploration of the available information at the conceptual level to the user of the portal. The portal has been designed to provide complete access to information related to Onto Web network to all its community members. This portal supports many features related to browsing, raising a query on the information stored in the portal, accession and provision to the stored content. Another interesting characteristic of this system lies in the way of its presentation of information to the users, while organizing the information. It creates distinction of class and properties of the concepts. Class categories include sub and super class whereas the properties are literal and non literal. This system permits the users to conceptually interact with the available information employing the concepts and relationships from ontology.

As a part of research work on recommended based approach for knowledge resources in Communities of Practice of Elearning (CoPEs), Lamia Berkani et al. [16] proposes a recommendation system which is based on the hybrid semantic IF. The research idea employs a multilevel filtering which is collaborative, social and semantic applied on the profiles for proposing recommendation of knowledge resources. In CoPE, the recommendation of knowledge resources is made to the member in situations like information retrieval, design of learning circumstances, new resource and new members who integrates the system, but this recommendation based approach has to be tested in real time. It has been envisaged to develop recommendation system and measure its aptness with the help of a learning community comprising of students from USTHB University in Algeria.

For content based retrieval of cultural objects (cases) the approached used by Govedarova et al. [17], involves characterization of metadata and use of domain ontology. This concept of ontology is used in CBR process to simplify the complicated, multi-relational case structures. In order to create a standard system some cases were composed consisting of different attributes with simple data types such as string, integer etc. To evaluate the vitality of any attribute in representing the concept of ontology a feature of concept data type supported by jCOLIBRI framework has been used. In this architecture the value of any attribute will correspond to the instances of the linked concept. Besides this, another feature of jCOLIBRI which has been of advantage is- its two layer organization of the case base. Here the meta data of the cases is created by observing the details of their physical representation in the Electronic Catalogue (Persistency media) and is stored in the case base (In-memory organization), the two layers of case base are connected via connectors. These connectors read the value stored in the data base and returns them to the application by assigning a value to the attribute.

Razmerita et al. [18] addressed aspects of ontology based user modeling and presented a generic ontology- based user modeling architecture, (OntobUM), application in the context of a KM (fig. 1). The authors used KM Systems, designed to allow users to access and utilize the enormous available data sources, information and knowledge in different forms to facilitate the process of knowledge creation and dissemination, along with continuous building of knowledge for those engaged in the process of knowledge management. The main contribution of this paper consists in: (1) identifying aspects of user modeling relevant to KMSs, (2) integrating them in a generic framework based on ontologies. They gave a concrete example of the use of the OntobUM framework in an ontology-based KMS, but this framework can be adapted to different ontology-aware environments. The user ontology is implemented using Semantic Web technology. It is structured on extended Information Management Systems Learner Information Package specifications. They have identified characteristics of the users that are relevant for a KMS under the behavior concept, but the largest part of the user ontology is generic and it could be reused in other application domains.

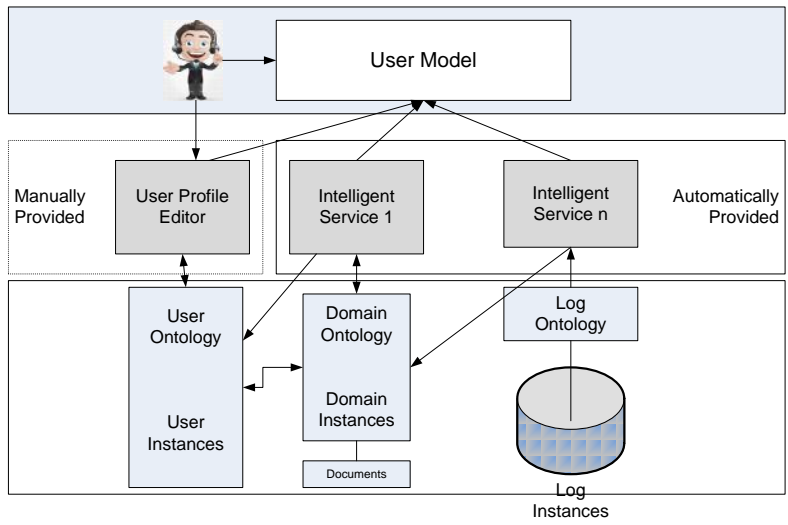

Fig 1: An ontology-based user modeling system [18]

The paper [19] proposes a hybrid approach of personalized Web Information Retrieval that utilizes ontology for retrieval of user's context, user profile that is temporarily updated based on users' browsing behavior and collaborative filtering for considering recommendation of similar users. Analysis concludes that Precision, Recall and F-Score of most of the queries for many users are improved with using the proposed method. Since the internet users typically make their queries, which contain one to three key terms so the retrieved results that are returned from the search engine may satisfy the general need but often fail to satisfy the user's search intention. Personalized search results satisfy the user's specific goal. This is the intent of the search. In this paper they presented a personalized search method by using ontology, dynamic user profiles, and collaborative filtering 
methods. Empirical analysis show significant improvement in precision.

L'ecu'e emphasizes on an approach that would serve the interest of the end users. In order to achieve this, a hybrid approach involving pure and classic collaborative-filtering methods and a semantic content-based has been presented. The former method involves automatic recommendation of services depending upon the users with similarity in their profiles, preferences and experience. The latter focuses on analysing the semantic similarity of services therefore it follows a Description Logic based reasoning [20].

Though this approach exhibits accuracy in recommendation of services to the users, but while taken into account the semantic expressivity i:e listing of redundant information on services description the same approach does not fetch good results. There exists some issues on scalability for future requirement in the first approach, while the second one is a time consuming phenomena as it involves DL reasoning which can be achieved by performing QCD computation through DL Difference, LCS and subsumption (even in ALE DL).

This article [22] is based on a survey which concentrates on the gap existing between the ontology research and its application in the mainstream $\mathrm{KM}$ including its business discipline. Though there is a huge demand of this ontology based technology in KM among the suppliers and consumers, but due to the hindrances like lack of communication and sufficient information, the gap still exists. Along with the persisting problem as described above, this article also talks about the developments taking place to address the problem; it says that the researches have been directed to show the significance and contribution of ontologies in the development of KM. The main contribution in the direction of synthesis of Ontology Technology and KM is the creation of a visual classification framework named as Semantic Web Matrix. This framework makes it easy to understand the business oriented account of application scenarios for ontology based KM.

A hybrid recommender system has been developed by Cantadoret known as News@ hand. It provides online news recommendation services using the semantic technologies. There are domain ontology concepts in the system which is used to describe the news items and user preferences. The system can support two models, a content-based model and a collaborative recommendation model. The choice of model depends upon the similarity between the item description and user profiles and semantics in the concepts involved. In this paper, a model has been evaluated which will take care of the users long-term interest as well as the current interest. For safeguarding the long- term interest of the user, a model has been developed which can personalize the order of visibility of news items, for protecting the current interest, a model which can reorder the news items has been developed [23].

Aldea et al. [24] describes a distributed KM system. The most important purpose of the platform is to enhance the industrial capabilities for monitoring, predicting the technological, resource and product related trends and challenges. The search and retrieval of information follows a two level approach in which ontology concept is used for specifying the query whereas the searching is done by a set of Internet agents, each representing the original ontology and performing a set of predefined roles. This architecture creates a system which can be easily updated as per future need. A key feature of this distributed KM system is that, it can be connected to the knowledge generation module of a KM system to fetch relevant information for the users from the web pages. But this is possible only after the search engine is completed and has undergone validation.

In [25], they addressed the main aspects of a semantic Web information retrieval system architecture trying to answer the requirements of the next-generation semantic Web user. An ontology and integrated intelligent system architecture for search operation support system and its implementation platform have been developed in this paper. The paper presented a system based in Ontology and Artificial Intelligent architecture for KM in the Seville Digital Library. It introduced a web-based CBR retrieval system which operates on an RDF file store. This system combines RDF representation and CBR recommendation methodology to do code selection for the resources codes; thus it applies a CBR approach with RDF data model. A prototype implementation that uses caching and fat operations is implemented and an intelligent agent was illustrated for assisting the user by suggesting improved ways to query the system on the ground of the resources in a Digital Library according to the preferences, which come to represent the interests. The test results show that the proposed service is a feasible solution that fields predictable performance in terms of response time and scalability. A crucial role in it plays the jColibri-based and Protégé components. They are the principal elements in the proposed architecture. Because jColibri is domain independent, and the domain-specific information for the system is captured entirely in the RDF ontology and ontology instances, the developed system could be easily transferred to other domains as well.

The study [26] explored the KM for library and information science (LIS). The study has done an online survey of KM education programs and an interdisciplinary approach of KM education is analyzed to find its linkage with LIS and also to examine the extent and scope of KM education with reference to LIS schools. The survey finds that the highest number of KM graduate programs originated from LIS followed by computing/engineering and business and management. The interdisciplinary nature of course names and KM skills enhance the KM implication in LIS education and library practices.

There are two main objectives of this survey [27], firstly it analysis the basic concepts of computer science used in representation of knowledge, simultaneously enumerating the effects associated with it. As per the survey, the concepts of ontology for knowledge representation can be classified into four categories. Static ontology, as the name suggests it describes the static world, a dynamic ontology which deals with the changing aspects of the world such as processes and transitions from one state to another, the third category is of Intentional ontologies, it takes into consideration the things in which the agents believe in while performing the search for a raised query which encompasses the wants, proves or disprove and arguments. Finally the last category is of social ontology. This takes into account the social settings authority, positions, roles, agents, permanent organizational structures or shifting networks of alliances and interdependencies. The second objective of the survey is to understand how the analysed techniques can be put to use theoretically and practically in information science.

The authors in [29] proposed two new families of inconsistency-tolerant semantics, that approximate the Consistent Query Answering (CQA) semantics from above and from below respectively, and converge to the CQA 
semantics in the limit. They studied the complexity of conjunctive query answering under the proposed two parameterized inconsistency-tolerant semantics, and showed a general result for all first-order rewritable ontology languages.

Fernández et al. [30] has proposed a semantic search model for better understanding of the semantic search problem. They studied the strong and weak points of the different proposals towards the semantic search paradigm. The work proposed the utilization of fine-grained domain ontologies to improve semantic retrieval in large repositories of unstructured information to address scalability, heterogeneity and usability challenges.

\section{ANALYSIS OF THE LITERATURE REVIEW AND OPEN PROBLEM PROPOSAL}

The literature indicates that so far the development of collaborative filtering based KM system either uses association analysis, data mining or machine learning like principle component analysis, artificial neural network but no ontology based collaborative filtering techniques have been used.

Ontologies are the one of the example of the new methodology for the system developments to implement knowledge. The purpose is to support knowledge sharing by providing common language. Artificial intelligence researchers have adopted ontologies as a comprehensive knowledge presentation to provide common sense reasoning in support of the knowledge tasks such as knowledge accomplishment and reuse. But there is a lack of dedicated techniques (Collaborative filtering based on the AI ontology) that can be used for efficiently managing the Research \& Development of the Science and Technology that can be transformed into intellectual capital. Therefore, the paper proposes a KM system

- To validate the deviation of the existing $\mathrm{KM}$ tool used in the corporate world and in the area of science \& technology.

- To develop the rationale on which intelligent tool is suitable for the area of science $\&$ technology.

- To develop various data flow diagrams with validation and incorporating it in the tool development.

- To develop a tool that can transform the exiting data available into knowledge.

- To examine the ontologies available and to validate the suitable ontology for the application of KM in science and technology.

\section{CONCLUSION}

In the realm of knowledge, KM supports to information systems technologies is progressing at a swift pace. The most important role of ontology in KM is to allow and to enhance knowledge sharing and reusing. In this paper, a view on ontology based on $\mathrm{KM}$ is presented. The main objective of the survey is to improve, understand and evaluate each approach by analyzing and using them. Based on the survey, we propose a technique on Collaborative filtering based on the AI ontology. In future, Ontology based collaborative filtering may lead researchers to a better enhancement in $\mathrm{KM}$ processing.

\section{REFERENCES}

[1] Fensel, D.; Ontologies: A Silver Bullet for KM and Electronic Commerce, Heidelberg, Germany, 2001

[2] Aldea, A.; Bañares-Alcántara, R.; Bocio, J.; Gramajo, J.; Isern, D.; Jiménez, J. and Kokossis, A.; Moreno, A., Riaño, D.: An ontology-based KM platform. In: Workshop on Information Integration on the Web (IIWEB 2003) at IJCAI 2003, pp. 177-182

[3] Ackerman, M. S.; McDonald, D.; Lutters, W.; and Marumatsu, J.; Recommenders for expertise management. In Workshop on Recommender Systems: Algorithms and Evaluation at SIGIR '99

[4] Pundt, H. and Bishr, Y., Domain Ontologies for Data Sharing-An Example from Environmental MonitoringUsing Field GIS, Computer \& Geosciences, 28, pp. 98-102, 1999

[5] Irene Polikoff, Ontology Tool Support,InTopQuadrant Technology Briefing, 2003

[6] Kougias I., Seremeti L., and Kalogeras D., OntologyBased KM in NGEEs, in Int. J. Pure Appl. Sci. Technol. 2(1) (2011), pp. 54-62

[7] Vallet, D.; Fernandez, M and Castells, P.,An OntologyBased Information Retrieval Model. In: The Semantic Web: Research and Applications 2nd European Semantic Web Conference (ESWC 2005), 29 May - 1 June 2005, Heraklion, Crete, Greece.

[8] Stojanovic, N., and Stojanovic, L.: Usage-oriented Evolution of Ontology-based KM Systems. In: Proceedings of the 1st Int'l Conf. on Ontologies, Databases and Application of Semantics, Irvine, CA (2002)

[9] Probst, G., Raub, R., Romhardt, K., and Doughty, H.: Managing Knowledge: Building Blocks for Success, Prentice Hall (1999)

[10] Decker, S., Erdmann, M., Fensel, D., and Studer, R.: Ontobroker: Ontology Based Access to Distributed and Semi-Structured Information. In R. Meersman et al., (eds.):DatabaseSemantics: Semantic Issues in Multimedia Systems, Kluwer Academic Publisher, 351369, 1999

[11] Barnaghi, P.;Wang, W. and Kurian, J.C.Semantic Association Analysis in Ontology-based Information Retrieval In: Handbook of Research on Digital Libraries: Design, Development, and Impact. IGI Global,2009, 131 - 141. ISBN 1599048795

[12] Aviv, S.; and Gal, A. Enhancing portability with multilingual ontology-based KMDecision Support Systems, 45 (3) ,2008, pp. 567-584 June

[13] R. Bañares-Alcántara, L. Jiménez, and A. Aldea, Multiagent systems for ontology-based information retrieval, In: Luis Puigjaner and Antonio Espuña, Editor(s), Computer Aided Chemical Engineering, Elsevier, 2005, Volume 20, Pages 1549-1554, ISSN 1570-7946, ISBN 9780444519870, 10.1016/S1570-7946(05)80100-2.

[14] Paralic, J. and Kostial I, Ontology-based Information 
Retrieval,In Proc. of the 14th International Conference on Information and Intelligent systems, IIS 2003, Varazdin, Croatia, September 2003, ISBN 953-6071-223 , pp. $23-28$.

[15] Jarrar, M., Majer, B., Meersman, R., Spyns, P., Studer, R., Sure, Y., and Volz, R. "Web Portal": Complete ontology and portal. OntoWeb Deliverable, 6, 2002.

[16] Berkani, L., Nouali, and O.,Chikh, A. A Recommendation-based Approach for Communities of Practice of E-learning.

[17] Govedarova, N., Stoyanov, S.,andPopchev, I. An ontology based CBR architecture for KM in BULCHINO catalogue. In Proceedings of the 9th International Conference on Computer Systems and Technologies and Workshop for PhD Students in Computing (p. 67). ACM, June, 2008

[18] Razmerita, L., Angehrn, A., andMaedche, A. Ontologybased user modeling for KM systems. In User Modeling 2003, pp. 213-217, Springer Berlin Heidelberg, 2003

[19] Mittal, N.;Nayak, R.;Govil,MC; and Jain,KC .; A Hybrid Approach of Personalized Web Information Retrieval, 2010 IEEE/WIC/ACM International Conference on Web Intelligence and Intelligent Agent Technology, P308-313

[20] L'ecu'e, F.; Combining Collaborative Filtering and Semantic Content-based Approaches to Recommend Web Services, 2010 IEEE Fourth International Conference on Semantic Computing, P 200-205.

[21] Egbu, C.; Kurul, and E.;Quintas, P.; Hutchinson, V.; AhmedC.A.; Al-Ghassani;Ruikar, K.; Techniques \& Technologies for KM Work Package 3- Interim Report, 2003

[22] Mika, P.; and Akkermans, H.; Towards a New Synthesis of Ontology Technology and KM Technical Report IRBI-001, Submittedto the Knowledge Engineering Review in April, 2004.
[23] Cantador, I.;Bellogín, A.; and Castells, P.; Ontologybased Personalised and Context-aware Recommendations of News Items, 2008 IEEE/WIC/ACM International Conference on Web Intelligence and Intelligent Agent Technology, P-562565.

[24] Aldea,A.; Banares-Alcantara,R.; Bocio,J.; ramajo,J.;Isern, D.; Kokossis, V;Jimenez, L.; Moreno,A.; and Riano, D. ; An Ontology-Based KM Platform, In Workshop on Information Integration on the Web (IIWEB'03) at IJCAI ,Vol. 3, pp. 177-182,2003.

[25] Martín,A.;and León, C.; Expert KM based on Ontology in a Digital Library, In ICEIS 2010 12th international conference on enterprise information systems, Madeira (Portugal), pp. 291-298, 2010.

[26] Roknuzzaman,Md.; andUmemoto, K.; KM's Relevance to Library and Information Science: An Interdisciplinary Approach, Journal of Information \&KM, Vol. 7, No. 4 279-290, 2008

[27] Jurisica, I.; Mylopoulos, J.; and Yu, E; Ontologies for KM: An Information Systems Perspective, Knowledge and Information Systems, 2004, 6: 380-401

[28] Koutkias, V., Bekiaris, E., Maglaveras, N., and Gkemou, M. Towards An Ontology-Based Model For User Profile Representation In A Cloud Infrastructure. International Journal of Electronic Commerce, 4(2)., 2013

[29] Bienvenu, M., and Rosati, R, Tractable approximations of consistent query answering for robust ontology-based data access. In Proceedings of the Twenty-Third international joint conference on Artificial Intelligence (pp. 775-781). AAAI Press., 2013

[30] Fernández, M., Cantador, I., López, V., Vallet, D., Castells, P., and Motta, E. Semantically enhanced Information Retrieval: an ontology-based approach. Web Semantics: Science, Services and Agents on the World Wide Web, 9(4), 434-452, 2011 